February - 2008

\title{
Exploring the Role of Distance Education in Fostering Equitable University Access for First Generation Students: A phenomenological survey
}

\author{
Lisa C. Priebe, Tamra L. Ross, and Karl W. Low \\ Athabasca University - Canada’s Open University
}

\begin{abstract}
Using a qualitative study of distance education (DE) learners whose parents have not accessed post-secondary education (PSE), this paper proposes themes for further research in the study of first-generation students (FGS). This survey asked a number of open-ended questions about parental influences on university enrollment, and respondents' reasons for choosing university in general and DE in particular. Findings were consistent with current research in many areas focusing on debt aversion, lower parental guidance, older starting age, and difficulty separating from familial roles. Differences were noted, including lower parental valuation of PSE and an increased emphasis on non-educational priorities, such as family and work. The limitations of the current study are discussed, as well as suggestions for future FGS research in DE.
\end{abstract}

Keywords: distance education; first generation students; education equity; post-secondary education

\section{Introduction}

Recent data has shown that a Canadian university focusing on distance and open education draws a much higher proportion of first generation students (FGS) - those whose parents have not completed post-secondary education - than do traditional universities in Canada. Nationally, about 51 percent of the student population in Canadian universities have parents who have not completed university (Drolet, 2005), while at Athabasca University, an open Canadian university specializing in distance education (DE), 72 percent of students have parents who have not completed university (Athabasca University, 2006).

When we began to investigate reasons for the higher proportion of FGS accessing distance education at Athabasca University, we found no comprehensive studies specifically aimed at explaining why DE populations might have a higher proportion of FGS. Therefore, a small qualitative study of FGS accessing distance education at a variety of universities was performed to gain insight into the reasons that FGS are selecting distance and open education, and to provide direction for future studies. Our goal was to begin to understand how DE influences enrollment of first generation students.

Studies in this area are limited, so two primary objectives were identified: 1) to determine potential factors that may explain why a higher proportion of FGS are choosing DE, and 2) to 
identify potential areas for future, detailed study. A qualitative, phenomenological approach was chosen to explore the lived experience of FGS enrolled in a distance learning environment.

FGS have been recognized as an underserved group in the Canadian post-secondary system. Statistics Canada has confirmed a strong correlation between parental education levels and participation in post-secondary education (Drolet, 2005). Though financial considerations are a major factor limiting university access for FGS, Drolet concludes that university participation is more strongly correlated with the level of education achieved by parents. Drolet's survey of 2001 university participation levels showed that only 16.6 percent of children with parents who had high school education or less attended university, compared with 27.8 percent of children whose parents had attended college, and 49.6 percent of children whose parents had completed university.

Several explanations for this inequity have been noted, including differences in how FGS and continuing generation students (CGS) - students whose parents have previously attended university - prepare for university. Parents of FGS are generally less involved in helping their children apply for university study because they are less informed about available programs and options, application processes, and the importance of early preparation (Astin \& Oseguera, 2004; Ceja, 2006; Hahs-Vaughn, 2004; McCarron \& Inkelas, 2006; Pascarella, Pierson, Wolniak \& Terenzini, 2004). This lack of input is a particular disadvantage to FGS who select traditional enrollment (full-time study, direct-entry from high school, etc.) (Hahs-Vaughn, 2004) or apply to highly selective institutions (Astin \& Oseguera, 2004). Aston and Oseguera (2004) found that students from highly educated families are three times more likely to attend a highly selective institution than students from families with a middle education level, and five times more likely than FGS.

Further, compared to CGS, FGS may experience higher anxiety about leaving home to study (London, 1989), changing family role assignments (Lohfink \& Paulsen, 2005; London, 1989) and incurring debt (Lohfink \& Paulsen, 2005). Cofer, Somers and Woodhouse (2004) reported that FGS are particularly debt averse, possibly due to limited knowledge of the student loan system. FGS may also experience incongruence between their family backgrounds and the social role of a post-secondary student; this highlights the importance of the home culture in preparing students for the adjustment to higher learning institutions (Hsiao, 1992; McCarron \& Inkelas, 2006).

Because FGS may delay entrance to university and take longer to complete a degree, they may also have to balance study with additional commitments, such as children (Hahs-Vaughn, 2004; McCarron \& Inkelas, 2006). In general, FGS work more hours while studying, and complete fewer credit hours per year of study (Hahs-Vaughn, 2004; Lohfink \& Paulsen, 2005; Pascarella et al., 2004). These factors, combined with the culture shock experienced by FGS entering the academic environment (Choy, 2001; Lohfink \& Paulsen, 2005; Pascarella, Wolniak, Pierson, \& Terenzini, 2003) and the lack of preparation for transitioning to university study (Hahs-Vaughn, 2004; McCarron \& Inkelas, 2006), may lead to the demonstrated higher attrition rates of FGS (Lohfink \& Paulsen, 2005; Choy, 2001; McCarron \& Inkelas, 2006). Choy (2001) found that the first year attrition rate for FGS was double that for CGS, which is of particular importance when paired with the observation that after the first year, the disadvantages affecting the school performance of FGS have less influence (Hahs-Vaughn, 2004).

Since FGS often delay their entry into the post-secondary system or suspend their studies for as long as several years (McCarron \& Inkelas, 2006), when they return to study, these FGS enter as adult learners with special needs that may not be addressed by a system designed for younger 
students. To better serve these students, McCarron and Inkelas (2006) suggest that universities provide supports for adult learners, which include part-time study options, enhanced counseling services, online course delivery, accelerated study options, and day care for children. All but the last of these are immediately addressed in most distance education environments. Additionally, Hahs-Vaughn (2004) notes that FGS are less likely than CGS to attend a college that is far from home (see also Lohfink \& Paulsen, 2005); this highlights the importance of accessibility for these learners.

\section{Participants}

\section{Research Methods}

For this study, FGS were defined as post-secondary distance education students or alumni whose parents had never attended a post-secondary institution or completed a postsecondary course or program. In addition, participants were of the age of majority for their geographic region; able to use and access email; fluent in English; and enrolled in or finished a post-secondary course or program delivered via DE. Research was not limited to students of any particular university. A survey was favoured over an interview format to ensure that the study could include students in all countries within budget and time constraints.

Advertising for the survey was placed in student discussion forums and publications with an emphasis on DE. In total, 15 individuals responded to the call for participants, of whom four did not return the preliminary eligibility questionnaire, and two were deemed ineligible because their parents had prior post-secondary experience. The remaining nine were provided with the full survey, of whom seven replied and were included in our sample. The sample was comprised of a self-selected group that had enrolled in, or completed courses or programs from, various universities via distance education. Five of the seven participants had accessed distance education at more than one university.

Due to the lack of any standard definition of the ideal sample size for qualitative research, we used as a guideline the theoretical saturation paradigm defined by Guest, Bunce and Johnson, whose 2006 study, "How Many Interviews Are Enough,” attempted to establish a standard for determining when theoretical saturation has occurred. Building on Morse's (1995) observation that "saturation is the key to excellent qualitative work" (cited in Guest, Bunce \& Johnson, 2006, p. 60), the team aimed to determine ideal sample sizes for qualitative studies. The researchers noted that while probabilistic sampling is the ideal method of performing research, "it is virtually impossible to do so in the field (Bernard 1995; Trotter \& Schensul 1998) . . . especially . . . for hard-to-reach, stigmatized, or hidden populations” (p. 61). Additionally, Romney, Weller and Batchelder (1986) applied a mathematical test to show that in anthropological studies of discrete communities, sample sizes as small as four can be sufficient to provide reliable results.

Building on these guidelines, as well as Neilsen and Landauer's (1993) mathematical saturation model based on the results of six qualitative studies (cited in Guest, Bunce \& Johnson, 2006, p. 78), Morse's contribution to the Handbook for Qualitative Research 
(Denzin \& Lincoln, 1994), and the team's own experiment, Guest, Bunce and Johnson concluded that theoretical saturation generally occurs in as few as twelve interviews, and that for "high-level, overarching themes ... a sample of six interviews may [be] sufficient to enable development of meaningful themes and useful interpretations" (p. 78).

Our study attempted to discover and evaluate a hidden population for the purpose of isolating major themes for possible future research; therefore, our sample of seven participants was deemed appropriate and in keeping with the guidelines for high-level qualitative research. To encourage participation, a cash prize of $\$ 150$ was offered and awarded to a participant selected by random draw.

\section{Materials}

An eligibility questionnaire and a 24-question, self-response survey were developed for this study. The survey included four sections: 1) general demographic information; 2) family educational history; 3) respondents' educational environment; and 4) personal feelings about distance education. Survey questions were designed to be open-ended where possible, and to elicit the description of personal feelings and experiences. The surveys were sent and returned via email and required the participant to have access to a computer, email, and an MS Word-compatible word processor.

\section{Procedure}

Potential participants were asked to contact the researchers via email to obtain an eligibility questionnaire to be returned by email one week prior to receiving the final survey. The eligibility questionnaires were screened, and ineligible individuals (those whose parents had prior post-secondary experience) were removed from the study. The final survey, including a detailed information sheet, was sent to participants with a deadline for submission set for three weeks following receipt of the survey. The information sheet notified participants that submission of the completed survey would grant consent to use the results, and that all submitted information would remain confidential. Returned surveys remained unopened until all responses were collected.

Each researcher received a copy of all surveys, without information identifying the participants, and individually analyzed the content of each survey to isolate common experiences and attitudes. Following this, the researchers discussed their impressions and developed theme groupings based on the responses. The researchers met a third time to review and revise the thematic groupings, with reference to the original surveys, to ensure that the themes remained consistent with participant responses. Finally, the themes were reviewed alongside relevant research to identify areas of congruence and new themes arising from the survey results. 


\section{Thematic Analysis}

The findings that follow are separated into four sections based on themed groupings identified from the survey responses. The first examines similarities in starting age and life commitments common to our respondents. The second examines the factors of parental assistance with university application, pre-conceived notions about university study, and debt aversion. The third focuses on work ethic, individuality, and discouragement. The fourth and final section considers the influence of social roles and expectations. Where these themes are congruent with available research, we have noted this. We have also noted several factors that require more study to determine if they are affecting enrollment and persistence for FGS.

\section{Starting Age and Commitments}

One result that was consistent on all responses related to starting age: all of the respondents had been out of high school for at least two years, and in some cases a decade or more, before beginning DE study. All who had entered the post-secondary system within two years of leaving high school had suspended their studies for several years before returning to serious study. All but one of our respondents had children and cited childcare responsibilities as among the factors leading to their choice of DE. One noted that DE allowed her to fulfill both academic and parental roles:

"I don’t have to give anything up to be a distance education student. I can study when my kids are at school, and put my books away when they come home. I can take a day to do housework or yardwork without missing a class or a deadline.”

Four of our participants also cited a reluctance to relinquish paid work to return to school, one noting that, "distance education gives me the opportunity to continue with my educational goals while continuing to work." All respondents indicated a strong desire to complete their education, and many noted that study was enjoyable, a luxury. Indeed, one student said he was “mad keen” on attending university.

If these findings hold true for a significant portion of the FGS selecting DE to complete university, it would suggest that, in general, FGS start university later and that their status as adult students with additional responsibilities is a significant factor in their choice of DE. McCarron and Inkelas's (2006) findings show not only that FGS often delay the start of their education, but also that many FGS do not complete their degrees within eight years of completing or leaving high school. Findings on attrition rates for FGS (Choy, 2001; Lohfink \& Paulsen, 2005; McCarron \& Inkelas, 2006) support our finding of the delayed completion of studies. What surprised us was the consistency of this finding, with no respondents beginning DE post-secondary study before the age of 20 .

The supposition that a later starting age correlates with a higher level of life commitments, and that these commitments are a significant factor leading to the choice of 
DE, is not directly supported. It follows from McCarron and Inkelas's (2006) recommendation, however, that universities can best serve adult learners by providing opportunities for part-time study, child care, and evening contact hours. While their recommendations referred only to traditional university, DE can address all of these issues by allowing parents to stay at home and employed students to arrange study around their work schedules. Indeed, this desire to balance study with other life commitments was noted by many of our respondents, such as the mother of a child with health problems who commented: "When my son is hospitalized, I know that the quiz I wanted to write can wait until next week and it alleviates [the stress] I'd have at a brick and mortar school." All but one of the respondents stated that they would not be completing their degrees without access to DE. The remaining respondent noted that if DE were not an available option, she would have attended university much later because she had a young family.

\section{Parental Assistance, Education Stigma, and Debt Aversion}

As expected from the literature review (Ceja, 2006; McCarron \& Inkelas, 2006), our cohort noted a distinct lack of parental guidance through the process of applying for university. Specifically, respondents noted that their parents were not versed in the processes of university application, program selection, and obtaining funding. A typical response to the question about parental guidance was that, "neither of them had any experience in that area. I think they were even more confused by the calendars than I was.”

Similarly, our survey also indicated a significant level of debt aversion as suggested by the literature (Cofer, Somers \& Woodhouse, 2004; Lohfink \& Paulsen, 2005). One respondent indicated:

"Distance education gives me the opportunity to continue with my educational goals while continuing to work. This enables me to pay for all my courses without any financial support ... [from] outside sources, which means I will not have a large dept [sic] load like some of my friends."

Other respondents also indicated that they chose DE so that they could continue to work while they studied.

Against our expectations, no respondents indicated a negative initial opinion of DE, nor did they feel their parents were disapproving of DE. Instead, both parents and students were generally more concerned with degree completion. One respondent indicated that DE would be better received by her family than traditional post-secondary education: "I knew that they'd react favourably toward distance education because it allowed me to take care of my son." Another stated that her parents had "no comment about my attending university via distance education, they were just happy I attended.” While our 
experience is that many students still consider DE to be unusual or non-traditional, it is possible that parents who lack university experience are less likely to pass on this stigma.

For the FGS accessing distance education, comments such as, "my parents never really expected me to pursue any post-secondary education," and "they neither encouraged nor discouraged me from post-secondary education, it was kind of left up to me to pursue or not," were common. Conversely, Ceja (2006) noted that the parents of his participants, who were all American Chicana students attending traditional university, had a feeling that post-secondary education was a necessity for their children. This contrasts with the general theme we found in our survey, which was that parents were generally supportive of the pursuit of post-secondary education, but not committed to ensuring that their children would pursue it.

\section{Work Ethic, Individuality, and Discouragement}

Four of our respondents demonstrated a strong work ethic and a perception that full-time, traditional study is self-indulgent. Many said it was critical that they support themselves through school. A single mother selected DE so she could be home with a sick child, but also stated:

"I learned a great deal about work ethic from my mother . . . it makes it difficult for me to NOT work. I think if my son's [health improved], I'd have a hard time allowing myself to stay in school instead of going back to work. It almost feels indulgent of me to pursue this education.”

She also indicated a reluctance to rely on welfare. Moreover, other respondents echoed her commitment to self-support, with one commenting "we were encouraged . . . but only if we could do it on our own financially." One individual stated that people unable to fund their own education should get jobs instead, while another rejected the option of part-time study at a traditional university to care for her child and study full-time via DE simultaneously.

This commitment to balancing education with other obligations may stem from values instilled by the parents of FGS. In addition to the respondent who said her parents would approve of DE because it allows her to care for her son while studying, another was offered assistance by her father, but refused, "unwilling to have him compromise his life for mine.” All respondents had high standards for work and scholastic achievement.

A related, but less prominent, theme is a preference for independent study. The two students who stated this most explicitly had parents with the least education of our cohort; in both cases, the parents had not completed primary school. The first of these, whose father was illiterate until shortly before retirement, recalled her experiences "as a young child having to find my own solutions to school problems because [my parents] did not have any education to help me.” She decided as a young child that she would eventually pursue post-secondary education. The other of these respondents noted that 
DE "suits my style of learning. Sitting in lectures taking notes all day was wasted time for me. I do not learn by listening and writing. I learn my reading and I prefer to do it on my own." She also noted a preference for working at her own pace.

Others noted a desire to accommodate learning to their lifestyle and to work on their "own terms." One likened DE to "a truly personal solo sailing trip around the world . . . a one-on-one encounter with the course materials gives me a more 'intimate,' less filtered comprehension of the subject matter.” Independence also appeared as factor encouraging a return to study, as one participant noted, "I didn't want to be someone's assistant anymore. I'm done working to put money in other people's wallets.”

One returning student said that her parents "did not see the need for [post-secondary]" and "never provided me any help emotionally, financially, or otherwise." Her mother believed she should stay home with the children, and she was also discouraged by her husband. Another respondent said her parents undermined her study plans:

"My father filled out all my high school forms, refused to fill out OSAP forms and ... effectively controlled me right out of a shot at university ... [if my parents knew I was in university I] suspect that they would see it as a waste of time and money."

For our participants, enrolling in university seems to be an act of empowerment, yet their continued commitments to parenthood and fiscal responsibility suggest that they have internalized the values instilled by their parents, but not the limitations. The aforementioned respondent characterizes her return to post-secondary, more than 20 years after completing high school:

"I want a degree. I want to know I can do it . . . doing higher education now is my way of shaking their negative influence . . . Their lack of education defined their world. My education will help to redefine my world.”

While some studies of the larger population of FGS indicate that parents of FGS are often emphatic about their children pursuing university (Ceja, 2006), in our cohort only one participant indicated strong parental support. This may suggest that a subset of FGS who have been discouraged from attending post-secondary may be accessing DE for its

distinction from traditional learning. Further, probabilistic research is needed to determine if such a pattern exists.

\section{Social Roles and Expectations}

Social roles and expectations consistently appeared to have a significant influence on the respondents' choices of post-secondary institution. These roles consisted primarily of parental expectations, but were coupled with self-expectations that may have stemmed in part from the respondents' upbringing and parental influences. It is important to note, 
however, that these social expectations were the perception of the individual respondents and do not imply any formal definition of class structure.

The first significant social expectation expressed by five of our participants was the prioritization of the family role over educational goals. One respondent said that while her parents did not overtly dissuade her from attending university, she was expected to prioritize her parental role:

"When I decided to begin post-secondary education my mother had asked me what the purpose was and she did not like it when I said I wanted more than just minimum wage jobs in my life, I wanted a career. She believed that I should stay home with my children. I did when they were younger and continued to study as well.”

Accordingly, she chose a distance learning model that enabled her to maintain both roles, thereby fulfilling educational goals while meeting parental expectations.

A second social expectation was a perception, real or perceived, of class boundaries and the notion that obtaining a university degree could propel one into a separate social “class.” In one respondent's words, “. . . they felt to go for university was to reach above one's station. I think their attitude justified my staying in my place for so very long.” This implies that the parental expectation to maintain a social "station" was a factor that delayed entry into the post-secondary environment. In addition, consistent with McCarron and Inkelas (2006), the respondent acquired additional responsibilities prior to her return to post-secondary learning, and the DE environment allowed her study without sacrificing family obligations. Another respondent noted that as a result of his higher education and new interests in art and politics, he and his parents have grown apart.

These results are consistent with London's (1989) finding that as young adults move toward increased independence; they also come into conflict with their established family roles. This dynamic leads to a complex interplay between the desire for personal growth through education, and the fear of losing the role in the family unit (London, 1989). Based upon the responses to our study, it appears that, for our cohort, this conflict is largely resolved through adopting a method of education that allows the student to compromise and develop multiple roles simultaneously.

\section{Limitations}

Due to the nature of phenomenological research, this study has several inherent limitations, particularly for the interpretation and application of our results to a larger FGS population. First, the number of participants was small and self-selected, and to date there has been very little research on the population characteristics of FGS DE students. Given this, it is important to reemphasize that this thematic analysis is intended to identify potential areas for future study, not to imply cause-and-effect relationships. Second, our analysis is subject to researcher interpretation, which is influenced by our 
personal distance learning experiences. To minimize the effect of individual bias, the researchers conducted individual thematic analyses prior to compiling the results. Themes that were not supported across multiple surveys, or that provided ambiguous interpretations, were discarded.

Additionally, our study did not differentiate between different modes of DE delivery. It is possible that these themes may or may not demonstrate a stronger association with one or more specific delivery options, such as correspondence learning, online learning, independent study, group or paced learning, and so on. Therefore, a multivariate analysis investigating FGS attitudes toward various delivery modes may help to clarify this issue. This study did not seek to identify the influence of cultural differences and, as a result, participation was limited culturally only by the respondents' ability to communicate fluently in English. Given that all but one respondent was located in Canada, with the exception that one respondent resided in Australia, it is prudent to assume that these results reflect a Western bias. Finally, our research does not attempt to compare FGS DE learners with DE learners as a whole. It is possible that the characteristics found in our population are common to the larger DE population. Further research to compare the common attitudes of DE learners with those of FGS DE learners specifically would be beneficial.

\section{Conclusion}

The one consistent factor revealed by our research was that the surveyed FGS are beginning university study at a later age than expected, and that they often suspend or delay their studies for long periods. Because current research does not follow these students throughout their lifetimes - McCarron and Inkelas (2006) report on rates of university completion only within eight years of the end of high school - it is not clear how many FGS are eventually completing their degrees, nor do we understand the factors that bring them back to school after a long delay.

Also while parental guidance for transitioning into post-secondary education was frequently cited as limited in our study, respondents did not provide sufficient information to explain how this parental limitation influenced their selection of DE. It is important, however, to note that none of the respondents indicated that their parents had pre-conceptions regarding DE, nor did they strongly encourage a specific delivery mode. Therefore, another potential area for research is whether parental bias, or lack thereof, regarding post-secondary delivery modes influences students' choices. Such a study might ask if parents who have attended a traditional university bias their children toward that delivery mode. In addition, the strong debt aversion cited by respondents who chose DE to remain self-supporting warrants additional study and raises questions about parental influence, lack of understanding of the student finance system, or a potential youth bias in the student loan system.

The independent learning style highlighted by some of our respondents may also provide insight into their selection of DE, given DE's emphasis on self-directed learning. The 
respondents were emphatic that DE provided the only option for them to incorporate university into their busy lifestyles, with all respondents indicating that they would be unlikely to complete their degrees without the option of distance delivery. Whether this individuality is unique to the study participants or a direct result of their FGS status is unknown, however. Therefore, it seems important to study how parents of FGS students may have influenced their development and attitudes, and whether a strong work ethic and individual learning style are correlated with familial influence and DE selection. Finally, we must determine if this ethic discourages FGS from leaving work to attend university.

The final theme, the influence of social role expectations, raised questions as to whether FGS are subject to unique social expectations that are correlated with traits such as adopting multiple commitments early in life, starting education at a later age, valuing a strong work ethic, and experiencing guilt when choosing to emphasize education over perceived social roles and responsibilities. As most respondents indicated a strong reluctance to let their education diminish their other roles, it seems important to determine if this relationship is correlated with FGS status and DE selection, or if it is common to post-secondary students.

\section{References}

Astin, A. W., \& Oseguera, L. (2004). The Declining “Equity” of American Higher Education. The Review of Higher Education, 27(3), 321-341.

Athabasca University. (2006). Report of Comparative Results of the Alberta Postsecondary Graduate Outcomes Survey: Class of 2003/ 2004. Institutional Studies. Retrieved June 13, 2007, from: http://intra.athabascau.ca/ois/grad_outcomes_2006.doc

Ceja, M. (2006). Understanding the Role of Parents and Siblings as Information Sources in the College Choice Process of Chicana Students. Journal of College Student Development, 47(1), 87-104.

Choy, S. (2001). U.S. Department of Education, National Center for Education Statistics, Students Whose Parents Did Not Go to College: Postsecondary access, persistence, and attainment. NCES 2001-126, Washington, DC.

Cofer, J., Somers, P., \& Woodhouse, S. (2004). Pushing the Boulder Uphill: The persistence of first-generation college Students. NASPA Journal, 41(3) 418-435. Retrieved June 10, 2007, from: http://publications.naspa.org/cgi/viewcontent.cgi?article=1353\&context=naspajou $\underline{\text { rnal }}$

Denzin, N. K., \& Lincoln, Y. S. (1994) Handbook of Qualitative Research. Thousand Oaks, CA.: Sage Publications. 
Drolet, M. (2005). Participation in Post-secondary Education in Canada: Has the role of parental income and education changed over the 1990s? Statistics Canada: Ottawa. Retrieved June 10, 2007, from: http://www.statcan.ca/english/research/11F0019MIE/11F0019MIE2005243.pdf

Guest, G., Bunce, A., \& Johnson, L. (2006). How Many Interviews Are Enough? An experiment with data saturation. Field Methods, 18(1), 58-82.

Hahs-Vaughn, D. (2004). The Impact of Parents' Education Level on College Students: An analysis using the beginning postsecondary students longitudinal study 199092/94. Journal of College Student Development, 45(5), 483-500.

Hsiao, K. P. (1992). First-generation college students (Report No. EDO-JC-00-04). Washington, DC.: Office of Education Research and Improvement. (ERIC Document Reproduction Service No. ED351079).

Lohfink, M. M., \& Paulsen, M. B. (2005). Comparing the Determinants of Persistence for First-Generation and Continuing-Generation Students. Journal of College Student Development, 46(4), 409-428.

London, H. B. (1989). Breaking Away: A study of first-generation college students and their families. American Journal of Education, 97(2), 144-170.

McCarron, G. P., \& Inkelas, K. K. (2006). The Gap between Educational Aspirations and Attainment for First-Generation College Students and the Role of Parental Involvement. Journal of College Student Development, 47(5), 534-549.

Pascarella, E. T., Pierson, C. T., Wolniak, G. C., \& Terenzini, P. T. (2004). First Generation College Students: Additional evidence on college experiences and outcomes. Journal of Higher Education, 75(3), 249-284.

Pascarella, E. T., Wolniak, G. C., Pierson, C. T., \& Terenzini, P. T. (2003). Experiences and Outcomes of First-Generation Students in Community Colleges. Journal of College Student Development, 44(3), 420-429.

Romney, A. K., Weller, S. C., \& Batchelder, W. H. (1986). Culture as Consensus: A theory of culture and informant accuracy. American Anthropologist, 88(2), 313338.

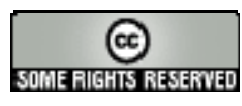

